\title{
Actividades generadoras de valor de las micro y pequeñas empresas metalmecánicas arequipeñas
}

\author{
Value-generating activities of micro and small \\ metalworking companies in Arequipa
}

\author{
Yezelia Cáceres Cabana \\ Universidad Nacional de San Agustín de Arequipa, Arequipa, Perú • ycacerescab@unsa.edu.pe \\ María Castillo Ureta \\ Universidad Católica San Pablo, Arequipa, Perú • mcastillo@ucsp.edu.pe \\ Gabriela Carpio Aconeira \\ Universidad Católica San Pablo, Arequipa, Perú • gabriela.carpio@ucsp.edu.pe \\ Juan Mardonio Rivera Medina \\ Universidad Continental, Arequipa, Perú • jrivera@continental.edu.pe
}

\section{Resumen}

El sector metalmecánico reviste particular importancia como parte de la cadena de valor de diversas industrias. El presente estudio contribuye al entendimiento del sector metalmecánico, mediante el análisis exploratorio de las actividades primarias y de apoyo que realizan las micro y pequeñas empresas (mypes) de dicho sector en la región Arequipa, Perú. El análisis se apoya en una encuesta a una muestra de 19 empresas y la aplicación de 73 entrevistas semiestructuradas a directivos del sector. Los resultados permiten la caracterización descriptiva de las mypes del sector, el análisis de las actividades de la cadena de valor, la identificación de factores de éxito y el reconocimiento de debilidades. El estudio identifica las actividades de la cadena que son fortaleza del sector metalmecánico y recomienda actividades vinculadas al diseño de optimización de planta y a las acreditaciones de calidad. Asimismo, se proponen recomendaciones para el fortalecimiento del sector.

Palabras clave: mypes, metalmecánica, competitividad, cadena de valor. 


\begin{abstract}
The metalworking sector is of particular importance as part of the value chain of various industries. This study contributes to the understanding of the metalworking sector, through the exploratory analysis of the primary and support activities carried out by micro and small companies (MSEs) of this sector in the Arequipa region, Peru. The analysis is supported by a survey of a sample of 19 companies and the application of 73 semi-structured interviews to managers of the metalworking sector. The results allow the descriptive characterization of the MSEs of the sector, the analysis of the activities of the value chain, the identification of both success factors and the recognition of weaknesses. The study identifies the activities of the chain that are a strength of the metalworking sector and recommends activities related to plant optimization design and quality accreditations. Recommendations are made for the advancement of the metalworking sector.
\end{abstract}

Keywords: MSEs, metalworking, competitiveness, value chain.

\title{
1. Introducción
}

Diversos estudios han evidenciado la importancia y contribución del sector metalmecánico en la economía de los países latinoamericanos. En Argentina el sector metalmecánico representó el 17\% del producto de la industria, en el año 2013; en Brasil, Colombia y México, al año 2012, el sector metalmecánico representó $27 \%$, $10 \%$ y $31 \%$ de la industria, respectivamente. Al año 2014, en estos mismos países, el sector metalmecánico participó en las exportaciones totales en las siguientes proporciones: Argentina, 15.7\%; Brasil, 15.8\%; Colombia, $3.1 \%$; y México, $62.3 \%$. Sin embargo, este sector atraviesa por escenarios muy complicados de competitividad. En términos de balanza comercial, China, el competidor global, pasó de un déficit de 15 mil millones de dólares en 2003 a un superávit de 350 mil millones de dólares en 2014, mientras que los países latinos indicados incrementaron significativamente sus importaciones de productos metalmecánicos procedentes de China: el valor de los productos importados se multiplicó diez veces en solo diez años, de 8 millones de dólares en el 2003 a 80 mil millones de dólares en el 2014 (Mendes de Paula, 2015).

Los gobiernos de la región han realizado inversiones para la construcción de capacidades mediante el fortalecimiento y generación de centros de innovación, conformación de clústeres y relacionados. De esta manera, han buscado lograr impacto en la competitividad empresarial que permita a los empresarios del sector acceder a herramientas para mejorar sus capacidades técnico-productivas, de gestión, así como fortalecer las redes empresariales y difundir las innovaciones.

En el Perú el sector metalmecánico es proveedor de bienes de capital como maquinaria, equipo e instalaciones para su utilización en los eslabones productivos de otros sectores, como minería, pesca, construcción y otros (SNI, 2019). En el sector metalmecánico operan más de 45 mil empresas formales, de las cuales el 98.7\% (44 918) son micro y pequeñas empresas (mypes) (Sunat, 2019). Asimismo, 1.5 millones 
de personas ejecutan actividades productivas relacionadas con la manufactura y el $62 \%$ permanece en la informalidad (INEI, 2019).

Las estadísticas revelan que es frecuente que las mypes tengan dificultades para mantenerse vigentes. Por ejemplo, durante el primer trimestre del 2018 se dieron de baja 37 mil 449 empresas (INEI, 2018). Si bien no se conocen las causas específicas de las bajas en las empresas, es posible que la gestión del valor tenga efectos concretos en la vigencia de los negocios. Cabe mencionar que el crecimiento en el Perú ha sido sostenido en los últimos veinte años; sin embargo, la productividad nacional no se ha incrementado en la misma proporción. Otros elementos que influyen en el sector son la informalidad empresarial, los problemas del marco regulatorio e institucional, las fallas en el comportamiento de los mercados de factores de bienes y servicios finales, acompañados por las debilidades de la gestión empresarial (García, 2012). A pesar de ello, la producción del sector metalmecánico en el Perú aumentó para el 2019 con un $10.7 \%$ (SNI, 2020).

El gobierno peruano ha identificado ocho ejes para impulsar la competitividad en los diferentes sectores productivos, incluyendo al sector industrial; los cuales son infraestructura, formación y acumulación de capital humano, mercado laboral, comercio exterior y cadenas logísticas, ciencia, tecnología e innovación, instituciones y ambiente de negocios (MEF, 2018). El sector metalmecánico es considerado como eslabón fundamental en la cadena de aprovisionamiento al sector minero. Por ello se vienen dando esfuerzos para la consolidación del clúster metalmecánico minero. Este esfuerzo de consolidación se da con mayor incidencia en el sur peruano, específicamente en la ciudad de Arequipa, debido a que en el entorno regional se encuentran ubicados importantes proyectos mineros. Se trata de un contexto relevante considerando las oportunidades que podrían tener los proveedores del sector metalmecánico en los encadenamientos productivos de diseño, producción y venta.

Sobre la base de lo expuesto, tomando a la ciudad de Arequipa como punto de referencia, este artículo tiene por objetivo describir y analizar los factores más relevantes que contribuyen a la generación de valor en las actividades primarias y de apoyo de las micro y pequeñas empresas del sector metalmecánico, además de sus principales subsectores, tales como fabricación de hierro y acero, construcciones metálicas, electromecánica y servicios técnico-industriales, y producción de máquinas y equipos. Para lograr este objetivo, en el presente estudio se aplicó un instrumento diseñado y validado para evaluar el desempeño de las actividades que integran la cadena de valor de pymes, descrito en Paravié et al. (2012).

La importancia de este análisis se centra en que los esfuerzos para mejorar los estándares de productividad y competitividad pueden estar localizados en las actividades cotidianas que se realizan en cada fase de los procesos de producción y comercialización, además de que es posible que el pequeño tamaño de las empresas pueda permitir que los cambios marginales sean más flexibles. Identificar las actividades que generan mayor valor y son fuente de competitividad puede contribuir a una mejor orientación para el fortalecimiento de las microempresas.

Este documento se divide en cuatro secciones. La primera muestra los fundamentos teóricos utilizados en el estudio; la segunda se enfoca en las herramientas metodológicas cualitativas 
y cuantitativas aplicadas; la tercera ofrece los resultados y hallazgos, detallando las características de las mypes, e identifica los factores generadores de valor. La cuarta sección presenta las conclusiones e implicancias de política pública. Al momento de la presentación del presente artículo, el contexto mundial ubica al sector en medio de la pandemia del COVID-19; los hallazgos permiten identificar la situación del sector antes de los efectos de la pandemia. Es probable que la situación de pandemia haya agravado las vulnerabilidades y puesto en evidencia nuevos retos.

\section{Revisión de literatura}

El considerar el análisis de la cadena de valor como marco teórico se sustenta en que permite distinguir cuáles son las características competitivas diferenciales a partir de los encadenamientos de valor desde los proveedores hasta los clientes. Se trata de analizar los eslabones que constituyen la cadena de valor, es decir, el conjunto de actividades en las diferentes etapas de producción (Kaplinsky y Morris, 2000). Estos eslabones o actividades varían en cantidad, según sea el sector productivo y pueden ser ejecutadas por una o más empresas (Kaplinsky, 2000). El concepto y los elementos de la cadena de valor pueden localizarse en diversos espacios geográficos. Cuatro dimensiones claves para una cadena de valor global son estructura insumo-producto, cobertura geográfica, gobernanza y marco institucional (Gereffi, 1999).

Siguiendo el modelo teórico de cadena de valor, considerado como un instrumento para identificar las actividades desarrolladas en una empresa, con el objetivo de generar valor para el cliente final, se indica que las actividades de valor se dividen en dos grupos conocidos: actividades primarias y actividades de apoyo. Las primarias intervienen en la creación física del producto, en su venta y transferencia al cliente, en la asistencia o servicio posterior a la venta, mientras que las de apoyo sirven de soporte a las primarias. El aporte de valor se da en ambos sentidos, puesto que, al dotar de materias primas, tecnología, recursos humanos y diversas funciones globales se van generando aportes mutuos (Porter, 1987).

Siguiendo a Paravié et al. (2012), para el análisis de los sectores industriales los procedimientos incluidos dentro de las actividades primarias son logística de entrada (que comprende la recepción, inventario y la distribución interna de los inputs del producto), operaciones (relacionadas a la transformación de las entradas en el producto final, instalaciones, técnicas de planificación, control de producción y de costos), logística de salida (relacionada a almacén e inventario y su distribución física a los compradores), marketing y ventas (relacionado al mercado, relación con el cliente, medios de promoción) y servicios (relacionado a la postventa, satisfacción y reclamos). Las actividades de apoyo son recursos humanos (puestos de trabajo, reclutamiento, capacitación, motivación, comunicación, trabajo en equipo), abastecimiento (disponibilidad de materia prima, gestión de compras, relación con proveedores), infraestructura (relacionada a la dirección general, la planificación, finanzas, contabilidad, asesoría legal, vinculados con las administraciones públicas y gestión de calidad) y desarrollo tecnológico (innovaciones y tecnología de la información y comunicación).

El enfoque de la cadena de valor propuesto por Porter (1987) es aplicado en la Guía general para el desarrollo de cadenas de valor sugerido por Nutz y Sievers (2016) para ser 
considerado como un marco de análisis para las pequeñas y medianas empresas de países emergentes. En este caso, el aporte específico referido a la utilidad del análisis de la cadena de valor se centra en (1) identificar las causas subyacentes de los cuellos de botella que impiden que la cadena de valor alcance determinadas metas económicas, (2) ayudar a comprender los incentivos de los agentes del mercado para contribuir a una solución, (3) señalar caminos hacia el cambio sostenible, centrándose en las oportunidades de mercado y (4) ayudar a identificar factores clave para las intervenciones.

Con relación al análisis de la ventaja competitiva, además de los sustentos de creación de valor, diversos autores (Peteraf, 1993; Prahalad y Hamel, 1990; Wernerfelt, 1984) se centran en la importancia de los procesos internos de las organizaciones y muestran que la ventaja de una empresa es menos dependiente de su posición en el mercado que de la gestión de recursos y capacidades internas exclusivas de cada organización; de esta manera, la evolución de enfoque de dirección estratégica y ventaja competitiva toma como pilares para la estrategia de las organizaciones los recursos, las competencias y capacidades organizativas centradas en procesos internos apoyados por la innovación.

Por su tangibilidad, los recursos engloban los de tipo financiero y físico. Recursos intangibles son los recursos humanos, marcas, patentes, vinculación con proveedores y clientes y la cultura organizacional. Estos influyen de manera importante en la creación del valor y la competitividad (Grant, 1996; Hall, 1992).

Como competencias esenciales para el logro de resultados de la organización se incluyen el saber, la experiencia acumulada, actitudes, habilidades, competencias, procesos (competencias tecnológicas, personales y organizativas). También es importante el aprendizaje colectivo como resultado de las interacciones con proveedores y clientes (Prahalad y Hamel, 1990).

Otros puntos vinculados directamente a la generación de valor son los indicadores de innovación y desarrollo tecnológico; estos frecuentemente guardan relación con sus capacidades y recursos. Así también es posible que la gestión de los procesos y procedimientos que conducen a la calidad se convierta en una estrategia competitiva (Labarca y García, 2011). Las capacidades descansan sobre los recursos, ya que las primeras contribuyen al incremento del stock de las segundas (Estrada, García y Sánchez, 2008).

El desarrollo tecnológico es importante en todas las áreas de la organización, prioritariamente en el proceso productivo, puesto que tiene un efecto directo en el mejoramiento del producto y proceso, ya que lo optimiza y reduce costos. Se logra a través de la implementación de acciones enfocadas en la innovación que se dan por la investigación y desarrollo que actualmente es llevada por el $42 \%$ de las empresas estudiadas. En esto también coincide la propuesta elaborada para el desarrollo del sector siderúrgico, metalmecánico y astillero en Colombia (Programa de Transformación Productiva, 2013).

La evidencia señala que los elementos de ventaja competitiva del sector metalmecánico son diversos: estándares de calidad; flexibilidad en términos del producto, volumen, maquinaria y procesos; capacidad de innovación; adecuados niveles de inversión; utilización eficiente de los productos; adición de características nuevas o deseables al producto, entre otros elementos 
competitivos presentes en el sector metalmecánico (Labarca y García, 2011).

El sector metalmecánico abarca distintos subsectores. El conocimiento del mercado, a través de investigaciones, permite a la empresa responder a necesidades específicas, y a su vez lograr la especialización productiva que le ayude a enfocarse en la elaboración y provisión de ciertos productos o servicios. La especialización productiva supone una mejora competitiva (Instituto de Desarrollo Industrial Tecnológico y de Servicios, 2005). Un elemento que contribuye a la especialización es la capacitación. En este sentido, conviene elaborar planes de formación y capacitación continuos alineados con los objetivos de la empresa: «apenas una de cada tres empresas del sector industrial lleva a cabo un proceso de producción eficiente»; descuidando también el manejo del marketing y ventas, debido al desconocimiento del mercado y las pocas estrategias de publicidad, promoción y comercialización implementadas (Saavedra y Milla, 2012).

La versatilidad con relación al tamaño de las empresas y su heterogeneidad en los procesos y procedimientos sugiere una oportunidad para la aplicación del enfoque de Porter (1987) para las mypes, sin dejar de considerar que en una empresa manufacturera es determinante la buena gestión del área de producción. En esta línea, para las empresas del sector metalmecánico sería conveniente contar con un diseño de planta eficiente y una filosofía de mejora continua. La tendencia sugiere resaltar los principales beneficios de la estandarización de los procesos, optimización de los tiempos, disminución de desperdicios, mejor utilización de recursos y reducción de los costos (Unión Industrial Argentina, 2013).

Respecto a las actividades de apoyo, sobresale la infraestructura relacionada con la capacidad de gestión y ello contribuye al crecimiento (Cohen y Ballara, 2012); otra de las actividades es el desarrollo tecnológico, donde aspectos como la innovación e investigación son fundamentales en las empresas (Saavedra y Milla, 2012).

Entre las actividades de apoyo destaca como importante el desarrollo tecnológico ya que, a través de la innovación, investigación y desarrollo, permite lograr a la empresa una ventaja en la diferenciación (Labarca y García, 2011) y los recursos humanos, donde aspectos como la motivación, formación y constante capacitación dentro de la organización pueden hacer la diferencia (Saavedra y Milla, 2012).

Además, el crecimiento escalar en los emprendimientos y los cambios marginales en la productividad agregada pueden surgir desde los eslabones más pequeños, conforme los actores del mercado fortalecen su posicionamiento competitivo empresarial, la capacidad de absorción del conocimiento, innovación, creatividad, recursos humanos y gestión desde los proveedores hasta los consumidores (Mathison, Gándara, Primera y García, 2007). En consecuencia, es posible que el tamaño de las empresas tenga relación con el acceso y uso de la tecnología, en particular para hacer más eficientes los procesos productivos.

\section{Metodología}

Sobre la aplicación del análisis metodológico a los diferentes sectores productivos, no existe un procedimiento estándar; asimismo, no necesariamente los procesos son secuenciales, dado que los procesos de análisis de la cadena de valor dependen de las condiciones y características propias de cada sector. También es preciso reconocer que la 
generación, pérdida y transformación del valor se da permanentemente. Bajo estas consideraciones se ha desarrollado un diseño metodológico de tipo exploratorio, descriptivo, no experimental, de corte transversal; el trabajo de campo combina enfoques cuantitativos y cualitativos. La técnica de muestreo es no probabilística, por conveniencia. La herramienta de acopio de información es mediante encuesta validada y publicada por Paravié, Rohvein, Urrutia, Roark y Ottogalli (2012), en un trabajo sobre la cadena de valor de pymes metalmecánicas. De otro lado, el método del análisis de las características de las actividades de la cadena de valor seguido en el presente estudio se deriva de la aplicación de un cuestionario integrado de preguntas cerradas y abiertas, considerando como elementos centrales de indagación las actividades primarias y de apoyo de la cadena de valor; se consulta sobre el tipo de recursos en el input de cada actividad y la gestión e influencia de estos en las actividades primarias y de apoyo.

La unidad de análisis corresponde a las mypes. De acuerdo con la definición peruana, las microempresas son aquellas cuyas ventas anuales llegan a 150 Unidades Impositivas Tributarias (siendo el valor de una UIT de 4200 soles para el año 2019 según Decreto Supremo $\mathrm{N}^{\circ} 298-2018-\mathrm{EF}$ ) y cuentan con 1 a 10 trabajadores; y las pequeñas empresas son aquellas cuyas ventas anuales llegan hasta 1700 UIT y pueden contar con 1 a 100 trabajadores según el Decreto Supremo DS 007-2008-TR (Ministerio de Trabajo y Promoción del Empleo, 2008). Sobre la base de este criterio, las mypes necesitan considerar y cumplir con ciertos parámetros: a) el número de trabajadores y b) el nivel de ventas anuales.

En la aplicación de las encuestas y entrevistas se excluyen a todas las empresas del sector que no cumplen con los criterios de una mype. Una limitación de los datos es que no permiten diferenciar o clasificar a las mypes, no solo por la productividad, sino también por las implicancias de otras variables en la gestión, tales como formalidad o informalidad.

\section{Tabla 1}

Actividades primarias de la cadena de valor

\begin{tabular}{lll}
\hline \multicolumn{1}{c}{ Actividades } & \multicolumn{1}{c}{ Recursos } & \multicolumn{1}{c}{ Elementos de análisis } \\
\hline Logística de entrada & $\begin{array}{l}\text { Control de entrada, } \\
\text { almacén, inventario y } \\
\text { control de costos. }\end{array}$ & $\begin{array}{l}\text { Metodologías de control y existencia de unidad de } \\
\text { almacenamiento, gestión de inventario y costos asocia- } \\
\text { dos al mantenimiento de inventario. }\end{array}$ \\
\hline & $\begin{array}{l}\text { Instalaciones, maquinarias } \\
\text { y equipos; técnicas de } \\
\text { planificación, control de } \\
\text { producción y control de } \\
\text { costos. }\end{array}$ & $\begin{array}{l}\text { Cambio en la demanda, uso de la capacidad instalada, } \\
\text { cuellos de botella, plan de mantenimiento, técnicas de } \\
\text { planeamiento y programación; diseño de producción, } \\
\text { control y seguimiento de calidad, detección de } \\
\text { desperdicios. }\end{array}$
\end{tabular}




$\begin{array}{lll}\text { Llmacén, inventario, } & \text { Espacio para almacenamiento, procedimientos de } \\ \text { Logística de salida } & \begin{array}{l}\text { manipulación de productos, gestión de almacén, } \\ \text { de distribución. }\end{array} & \begin{array}{l}\text { costos de distribución y mantenimiento, medios de } \\ \text { transporte y servicio de entrega de productos. }\end{array}\end{array}$

Mercado, relación con Identificación e investigación de mercados;

Marketing y venta el cliente y medio de identificación de necesidades y clientes; campañas producción.

publicitarias, página web, promoción de ventas.

$\begin{array}{lll} & \text { Postventa, satisfacción y } & \text { Servicio postventa, garantías formales, instalación de } \\ \text { Servicio } & \text { producto, atención al cliente, reclamos, registro de } \\ & \text { reclamos y acciones de mejoras. }\end{array}$

Nota. Adaptado de Paravié et al., 2012.

En la tabla 1 se muestran los componentes de la cadena de valor correspondientes a las actividades primarias, los mismos que están relacionados a los tipos de recursos con sus respectivos elementos de análisis y los componentes sustanciales del cuestionario por cada tipo de actividad primaria.

En la tabla 2 se muestran los componentes de la cadena de valor correspondientes a las actividades primarias, los mismos que están relacionados a los tipos de recursos con sus respectivos elementos de análisis y los componentes sustanciales del cuestionario por cada tipo de actividad de apoyo.

\section{Tabla 2}

\section{Actividades de apoyo de la cadena de valor}

\begin{tabular}{lll}
\hline Actividades & Recursos & Elementos de análisis \\
\hline
\end{tabular}

$\begin{array}{ll} & \text { Disponibilidad de materia } \\ \text { Abastecimiento } & \text { prima, gestión de compras } \\ & \text { y relación con proveedores. }\end{array}$

Cambio en la demanda, uso de la capacidad instalada, cuellos de botella, plan de mantenimiento, técnicas de planeamiento y programación; diseño de producción, control y seguimiento de calidad, detección de desperdicios. 


\begin{abstract}
Estructura, planeación, gestión de calidad,

Infraestructura inversiones, gestión de seguridad y gestión ambiental.
\end{abstract}

Organigrama, definición y conocimientos de objetivos, revisión de objetivos, política de calidad, documentación, identificación y control de procesos, certificación de calidad, auditoría interna, plan de inversiones, evaluación financiera, higiene, área de descanso, equipos de protección personal, impacto en medio ambiente, acciones correctivas.
Desarrollo tecnológico

Innovaciones, TIC y sistema de información.
Innovaciones, tipos de innovación, investigación y desarrollo, dificultades de innovación, disponibilidad de correo electrónico, acceso a internet y existencia de sistema de información.

Nota. Adaptado de la guía metodológica Paravié.

Los valores estimados fueron confrontados con las escalas de valoración por nivel de desempeño obtenidos para cada actividad, sea primaria o de apoyo, como se establece en la tabla 3 , mientras que el desempeño promedio (competitividad) fue llevado a comparación con la escala establecida en la tabla 4.

Los instrumentos de investigación se aplicaron a una muestra no probabilista, por conveniencia. Los entrevistados fueron 19 dueños de mypes de metalmecánica localizadas en Arequipa, dedicadas a subsectores como fabricación de hierro y acero, construcciones metálicas, electromecánica y servicios técnico-industriales, producción de máquinas y equipos.

\section{Tabla 3}

Escala de valoración por actividad de valor

\begin{tabular}{cc}
\hline Nivel de desempeño de la actividad & Valor \\
\hline Muy alto & $0.81-1.00$ \\
Alto & $0.61-0.80$ \\
Mediano & $0.41-0.60$ \\
Bajo & $0.21-0.40$ \\
Muy bajo & $0.00-0.20$ \\
\hline
\end{tabular}

Nota. Elaboración propia. 
Tabla 4

Escala de valoración global

\begin{tabular}{lc}
\hline \multicolumn{1}{c}{ Nivel promedio (competitividad) } & Valor \\
\hline Muy alta & $0.81-1.00$ \\
Alta & $0.61-0.80$ \\
Mediana & $0.41-0.60$ \\
Baja & $0.21-0.40$ \\
Muy baja & $0.00-0.20$ \\
\hline
\end{tabular}

Nota. Elaboración propia.

Para enriquecer el análisis de los datos obtenidos con las encuestas, se desarrollaron 73 entrevistas semiestructuradas en profundidad; el contenido de las preguntas orientadoras se estableció sobre los mismos elementos considerados en los cuestionarios.

\section{Resultados}

Los resultados del análisis de la generación de valor en las actividades primarias y de apoyo de las micro y pequeñas empresas del sector metalmecánico se dividen en las siguientes partes: la primera describe los resultados de las características de los empresarios de las mypes del sector metalmecánica en Arequipa, la segunda parte revela los resultados relacionados a la identificación de actividades primarias y de apoyo de la cadena de valor detallando el estado de los mismos en términos de escala de desempeño individual y global (competitividad), cuyos resultados se muestran en la tabla 5, y la tercera parte contiene los resultados de la identificación de los factores clave para las intervenciones en el sector.

\subsection{Descripción de los empresarios del sector metalmecánica}

Sobre las características los dueños de las mypes encuestadas del sector metalmecánica, que son las unidades de análisis, resaltan que son generalmente hombres cuyas edades oscilan entre 31 y 50 años mayoritariamente. Asimismo, el 26\% de las empresas que predominan en el mercado son las que tienen antigüedad de fundación de 6 a 10 años. En 
Tabla 5

Nivel de desempeño por cada actividad y del promedio de las actividades

\begin{tabular}{lcc}
\hline \multicolumn{1}{c}{ Actividades primarias y de apoyo } & Puntaje & Nivel de desempeño \\
\hline Logística de entrada & 0.66 & Alto \\
Operaciones & 0.65 & Alto \\
Logística de salida & 0.76 & Alto \\
Marketing y ventas & 0.60 & Medio \\
Servicios & 0.80 & Alto \\
Recursos humanos & 0.73 & Alto \\
Abastecimiento & 0.69 & Alto \\
Infraestructura & 0.55 & Medio \\
Desarrollo tecnológico & 0.58 & Medio \\
\hline Promedio nivel de desempeño & 0.67 & Nivel Alto \\
\hline
\end{tabular}

Nota. Elaboración propia.

algunos casos, los emprendedores continúan el negocio familiar, como lo señala la siguiente declaración: "Mi impulso fue cuando era joven. Mi padre se dedicaba a la herrería y, cuando vine a Arequipa, estudié mecánica de producción y empecé a trabajar en este campo de metalmecánica. Empecé primero con prácticas del instituto. Una vez tuve más experiencia, trabajé en talleres ya propios del campo» (MYPE $\left.\mathrm{N}^{\circ} 1\right)$.

En principio, dependiendo de la actividad a realizar, se requiere una mediana inversión de capital, que consiste en maquinarias básicas (cortadoras, soldadoras, otros) para iniciar los emprendimientos en este sector. No obstante, conforme los procesos son más complejos, se puede requerir mayor especialización e inversión fija. Cabe mencionar que en Arequipa los subsectores con mayor participación son fabricación de hierro y acero, construcciones metálicas, así como electromecánica y servicios técnico-industriales.

Del total de empresas analizadas, el 50\% son de los subsectores de fabricación de productos de hierro, acero y construcciones metálicas; el 38\%, de electromecánica, servicios técnicos industriales y producción de máquinas y equipos; el $12 \%$, de la industria automotriz y equipo para el transporte y actividades de preparación de productos para la industria metalmecánica. El 50\% está en la categoría microempresa y el otro $50 \%$, en pequeñas empresas. Del total de estas empresas de diversos subsectores y categorías, el 58\% son de propiedad familiar, frente a un $42 \%$ que no lo son; este punto es importante con relación a la cultura organizacional y toma de decisiones. 


\subsection{Identificación de la generación de valor en las actividades primarias y de apoyo}

Los resultados relacionados con la identificación de aspectos que impiden que la cadena de valor alcance determinadas metas económicas se organizarán en función de cada actividad primaria y de apoyo; por ello se encuentran en primer orden los recursos relacionados con la actividad de logística de entrada, donde el promedio de los ítems medidos obtuvo un puntaje de 0.66 sobre 1 , lo cual expresa un desempeño alto, debido a contribuciones relevantes por gestión de los recursos de almacenes e inventarios. Ello se corrobora con la entrevista donde un empresario señala: «Siempre sobra el material, cuando compramos siempre queda. Lo guardamos en un almacén y ahi queda para otro trabajo, se revende fierros que ya no se pueden utilizar. Lo que son planchas y acero en 36, grosor espesor de 3-16, un cuarto, 5-16 y tres octavos; es lo más comercial» (MYPE $\left.\mathrm{N}^{\circ} 2\right)$.

\subsubsection{Identificación de la generación de valor en las actividades primarias}

Puede darse el caso de que las empresas trasladen la ejecución de los proyectos solicitados a las instalaciones del usuario. Esto es frecuente en proyectos de mayor tamaño. En estas circunstancias, es posible que los almacenes se improvisen en espacios destinados para la gestión de insumos y materias primas, y que los productos se adquieran en función de los procesos y procedimientos previstos antes de la compra. Ello contribuye a economizar los insumos adquiridos. No obstante, se trata de una debilidad no tener control de entrada de los bienes adquiridos y no tener registros de movimientos de almacén.

Por otro lado, la actividad primaria denominada «Operaciones», en la calificación de un máximo de 1 , se puntuó con 0.65 , lo que corresponde a un nivel de desempeño alto para esta actividad estratégica. A este resultado contribuyen significativamente la gestión de los recursos de control de producción y costos, y de planificación, maquinarias y equipos. Esta actividad demanda el mayor presupuesto. La mayoría de las organizaciones carecen de diseño óptimo de planta y estandarización de procesos, por lo que coexisten con la capacidad ociosa y cuellos de botella. De igual manera, es importante notar que esta actividad inicia con la planificación del proyecto a desarrollarse, que tiene relación directa con la experiencia del empresario. El control de costos directos de fabricación es un asunto muy analizado según la totalidad de encuestados, como parte de los procesos de planeación básicos. "Aunque se planifica, el jefe de producción estima un tiempo, pero a la hora de ejecutar el servicio se toma a veces más tiempo, el dueño dice un tiempo y en realidad es otro» (MYPE $\left.\mathrm{N}^{\circ} 3\right)$.

Es posible que debido a la fluctuación de la demanda se requiera la organización de la producción, maquinaria y equipos, e incluso se presenten periodos donde hay capacidad instalada ociosa. Es posible también identificar limitaciones en la gestión para la adquisición de insumos a tiempo, en el espacio y diseño de planta, así como en los métodos de producción y la disponibilidad de personal adicional temporal. «El espacio que tengo es pequeño y no puedo realizar todos los trabajos que quisiera» (MYPE $\left.\mathrm{N}^{\circ} 4\right)$.

También otro empresario manifiesta que el área de producción se acomoda a las operaciones que demandan los clientes. "Todos los proyectos que tomamos están fuera del taller, mi taller es prácticamente un almacén, de todas maneras, tenemos un área de 
producción libre para cualquier eventuali$d a d »\left(\right.$ MYPE $\left.\mathrm{N}^{\circ} 5\right)$.

Sin duda, los aspectos mencionados afectan a la productividad y calidad de los productos, pero no son los únicos; cabe mencionar que, aunque se realiza control en la producción en el producto final, en la mayoría de los casos no se consideran controles en los procesos intermedios y no se documentan estos controles.

Otros aspectos importantes para las operaciones son los relacionados con el mantenimiento correctivo de la maquinaria y la gestión de la calidad. En este aspecto hay avances en las mypes proveedoras de las empresas industriales. «Sí, las empresas de Supermix, Gloria y Yura nos contratan, nos estamos abriendo en esos campos. Directamente con los dueños, no; pero con los ingenieros que están encargados del mantenimiento de las empresas, si se tiene una pequeña reunión. Ha habido el caso del encargado que viene y hace una inspección de la producción. En cuestión de servicios, lo manejan todo con el jefe de producción; en cuestión de costos, un informe técnico de lo que se ha elaborado. Tratamos de atender el $100 \%$ de lo que pide el cliente; no se ha hecho una investigación de mercado, pero quizás de los competidores que brindan el mismo servicio tratamos de sacar información y se compite en precios» (MYPE N 6).

También se destaca que los procesos que contribuyen en mayor medida a la gestión de los recursos de la actividad de operación están dados por factores como el diseño, soldadura y montaje, procesos que motivan a la adquisición de tecnología del extranjero para optimizarlos. «Por el área de la soldadura sería interesante ver algo nuevo, una feria tecnológica que tenga el tema de unión de materiales porque, por ejemplo, ahora $3 \mathrm{~m}$ te vende un líquido que puedes poner en tu tubo que tiene un aditivo, lo pones a tu base y queda fijo, tan fuerte como una soldadura. El tema es unión de materiales» (MYPE $\mathrm{N}^{\circ} 7$ ).

En este sentido, podría considerarse que el conocimiento empírico y la limitada información del mercado perjudican el aprovechamiento de oportunidades de venta. Ello podría confirmar la relación entre conocimiento y la definición del producto o servicio que se ofertará al mercado e información sobre la competencia. La adaptación de su oferta operativa, de su planta e instalaciones puede expresarse de la siguiente manera: «Nuestro plus competitivo está en que no nos cerramos a solo una cosa, por ejemplo, producir tanques; en realidad, nosotros empezamos haciendo tuberías, después hemos pasado a hacer tanques, después a hacer partes eléctricas, automatizaciones y así. En realidad, es variado. Como te digo, las soluciones que le damos a los clientes son múltiples» (MYPE $\mathrm{N}^{\circ} 8$ ).

De otro lado, en el recurso de control relacionado con factores de calidad en el proceso productivo y en el producto, el $53 \%$ de las empresas realizan una constante evaluación y aseguramiento de la calidad registrada; y se llevan a cabo a solicitud del cliente. Se manifiesta, como necesidad del sector, la implementación de sistemas de certificación de calidad por producto o proceso, lo que permitirá la mejora en la gestión de la calidad y en la capacidad productiva. «Nuestra marca es nuestra mejor recomendación; la responsabilidad y puntualidad es lo que más le gusta al cliente, sobre todo la eficiencia y control de calidad. Nosotros hacemos control de calidad, aunque no se tiene ficha técnica» (MYPE $\mathrm{N}^{\circ}$ 9). 
Otro empresario menciona lo siguiente: «La calidad en cómo se realizan las operaciones es muy valorada: tenemos un dossier de calidad y ese dossier es como la partida de nacimiento de cualquier cosa que vayas a hacer. Para eso necesitas desde tu materia prima, o sea de qué está hecha tu material, tu acero o si tienes la estructura, la composición adecuada o no para lo que vas a hacer, quién te lo soldó, cómo lo soldaste o qué proceso, qué dimensiones, todo es una ficha técnica, como la partida de nacimiento del producto que estás entregando» (MYPE $\left.\mathrm{N}^{\circ} 10\right)$.

Complementariamente, los aportes de los diagnósticos del sector evidencian que, para la consolidación de las mypes peruanas, es relevante considerar los costos de energía y la falta de asistencia para el acceso a los mercados (Ministerio de la Producción, 2015).

En la actividad primaria de logística de salida, para la cual se incluye el análisis de factores relacionados con almacén de productos terminados —el cual comprende productos de forma estandarizada que solo aplica a un tercio del total de las empresas en estudio-, en general las empresas del sector solo producen a demanda o requerimiento del cliente. Un empresario indica: «Nosotros, al realizar una venta anticipada, estamos en contacto con el cliente sobre su necesidady hacemos un papeleo como contrato para la venta; una vez hecha, pactamos el tiempo de entrega y entonces ellos finalizan con el pago y se les hace entrega del servicio de calidad» (MYPE $\left.\mathrm{N}^{\circ} 11\right)$.

Entre los aspectos que forman parte de los recursos de la logística de salida están los costos (de mantenimiento y logísticos); sobre este punto, resalta que los factores vinculados a costos y medios de distribución generalmente han sido resueltos al momento de la firma de contrato por el empresario.

Referido a la actividad primaria de marketing y ventas, de un máximo de 1 se puntuó con 0.60 , lo que corresponde a un nivel de desempeño medio. Se percibe que los empresarios tienen un conocimiento del recurso mercado, con información de los clientes, incluso es posible que hayan brindado varios servicios. Sin embargo, se aprecia que hay una brecha en la comunicación permanente y el afianzamiento con los medios de promoción y el uso de medios virtuales, páginas web u otros medios relacionados.

Dentro de esta misma actividad primaria, para los empresarios que desean ampliar su producción, se requiere mejorar su capacidad para reaccionar a los cambios del mercado mediante una fuerza de venta especializada en los procesos mecánicos y características de los bienes finales: «Lo que me cuesta más es conseguir personal para ventas. Puedes conseguir logísticos, soldadores, ayudantes, pero no hay personas para ventas. Un electricista, un mecánico conoce, él lo puede explicar más; se requiere que el vendedor cierre el trato con el cliente. Lo que encamina y hace que la empresa siga adelante son las ventas» (MYPE $\left.\mathrm{N}^{\circ} 12\right)$.

Referido a la actividad primaria de Servicios, de un máximo de 1 se puntuó con 0.80 , lo que corresponde a un nivel de desempeño alto, donde es destacable el involucramiento de todas las empresas en los servicios de postventa, como parte de la garantía formal; asimismo, la asistencia en los servicios de instalación, mantenimiento y reparación, lo 
cual es una fortaleza que aprovechan para la sostenibilidad de sus actividades económicas.

\subsubsection{Identificación de la generación de valor en las actividades de apoyo}

Sobre el nivel de desarrollo de las actividades de apoyo de la cadena de valor, iniciaremos por la actividad de Recursos Humanos. Como se muestra en la tabla 5, de un máximo de 1 , se puntuó con 0.73 , lo cual es considerado un nivel de desempeño alto. Aunque el desempeño de los recursos humanos es alto con relación al cumplimiento de gestión de recursos, también hay factores por mejorar con relación a los recursos. Por ejemplo, las capacitaciones sobre la seguridad en el trabajo carecen de evidencias objetivas, como resultado de monitoreo permanente de su cumplimiento y el manejo de manuales de seguridad e higiene. De igual forma, no se evidencian manuales sobre las funciones y responsabilidades por puesto de trabajo. Cabe mencionar que, en muchas empresas, se contratan trabajadores adicionales temporales en función de la necesidad circunstancial; en este sentido, son muy bajas las posibilidades de implementar acciones relacionadas con motivación, capacitación y compartir con el personal más estable.

En la actividad de apoyo de Abastecimiento, de un máximo de 1 se puntuó con 0.69 , como se aprecia en la tabla 5, el cual es considerado como un nivel de desempeño alto; los recursos analizados, que se muestran como mejor gestionados, son los referidos a la relación con los proveedores, donde el $89 \%$ de las empresas cumplen con los requisitos que se demanda. De igual manera, la gestión de disponibilidad de materia prima es destacable; con relación a este punto, se percibe la necesidad de mejoras en la gestión de compras. Las debilidades de coordinación y políticas de compra de insumos impactan negativamente en el proceso productivo; este problema es amortiguado por la ventaja del sector en cuanto a la disponibilidad de materiales e insumos y la relación con proveedores que apoyan con capacitaciones técnicas.

Con relación a la actividad de apoyo de Infraestructura, de un máximo de 1 se puntuó con 0.55 , lo que corresponde a un nivel de desempeño medio. Entre los recursos que destacan como mejor gestionados están los de estructura organizacional, objetivos y gestión de calidad. En cambio, se observan debilidades en la gestión de finanzas y de seguridad e higiene. Sobre la relevancia en la gestión de estos recursos en la empresa, se aprecia que la dirección empresarial es potestad del dueño, con los servicios de un contador para la declaración de impuestos. El enfoque es en el corto plazo, con orientación a la mejora en aspectos de la producción y calidad. Se reconoce la necesidad de certificar las prácticas de manufactura y documentar las acciones que se dan para la mejora de la calidad. Es posible que en la gestión de las finanzas no se observe un análisis riguroso. Como lo muestra la declaración del empresario: "Como es una empresa familiar, administramos junto con mi esposa y a la vez nos hacemos orientar con un profesional de contabilidad para los papeleos de la Sunat, etc. (MYPE N 13).

Otro empresario indica: «Yo soy el dueño $y$ tengo el apoyo con una contadora. El impacto principal es el derrame de líquidos, aceite que usan máquinas roladoras, grasa que se usa para deslizar los tubos» (MYPE N 14).

Con relación a la actividad de apoyo de Desarrollo Tecnológico, de un máximo de 1 se puntuó con 0.58 , como se aprecia en la tabla 5, el cual es considerado como un 
nivel de desempeño medio. Dentro de esta actividad, muy pocos recursos de investigación y desarrollo tecnológico se articulan con centros de investigación para el sector; se nota que las empresas realizan mejoras continuas en sus productos y procesos productivos o gestión organizacional mediante el uso de software, pero no ejecutan innovaciones, a excepción del $20 \%$ que están motivadas por la demanda. "Nosotros empezamos en líneas inoxidables. Me acuerdo que tenía el requerimiento de las tuberías y todas eran galvanizadas, pero con los años ya estaban todas corroídas y era cambiar a tuberías inoxidables. Luego vino un problema con un tanque, ¿cómo se puede solucionar? ¡Nos documentamos aqui en la oficina, al cliente le dimos la solución y quedó OK! También ese es el plus de la ventaja, porque incorporas nuevos procesos y lo hace cada vez más optimizados» (MYPE $\left.\mathrm{N}^{\circ} 15\right)$.

Cabe mencionar que las empresas proveedoras del sector minero han hecho esfuerzos en encaminarse hacia la certificación de sus estándares de calidad. Otras de la misma cadena se convierten en contratistas, es decir, son parte de la cadena en eslabones menores.

El definir las estrategias de promoción, publicidad y comercialización, además de evaluar su impacto en la empresa, permite posicionar sus servicios o productos en el mercado. Un aspecto relevante es que los plazos de pago para mypes del sector pueden hacer la diferencia entre la provisión o no del servicio, por su limitado capital de trabajo; por lo mismo, los esfuerzos en innovación son limitados.

Un alto nivel de productividad de los trabajadores en las empresas del sector es vital para el buen posicionamiento de las mismas en el mercado. Por ello, sería importante que cuenten con una formación que responda a las necesidades del sector, que podría ser brindada por los diversos centros superiores de la región, como indica la empresa: "Tan importante como la capacitación son los buenos hábitos en los empleados. [Es importante la disciplina, la responsabilidad del personal y que superen los exámenes médicos]» (MYPE $\left.\mathrm{N}^{\circ} 16\right)$.

Otra empresaria menciona: "La mano de obra es cara y mala, los operadores no están capacitados. Si el trabajador es homologado debe pasar la prueba. Es como una prehomologación. Solo la prueba cuesta 400 soles. Si vienen diez personas a postular, son 4000 soles, y si solo pasan dos, a veces por baratear uno tiende a buscar a cualquier personal» (MYPE $\mathrm{N}^{\circ} 17$ ).

\subsection{Identificación de gestión de recursos comunes generadores de valor}

Para las mypes estudiadas del sector metalmecánico - que, a su vez, comprenden especializaciones en subsectores de fabricación de hierro y acero, construcciones metálicas, electromecánica y servicios técnico-industriales y producción de máquinas y equipos-, se aprecia que las razones de generación de valor están centradas en factores específicos como los de procesos productivos (diseño, soldadura y montaje), acompañado de la gestión de proveedores mediante la provisión de insumos a tiempo, así como de los almacenes e inventarios que, en suma, contribuyen directamente al éxito de los procesos productivos; resalta que los proveedores suelen importar gran parte de los insumos, maquinaria y tecnología desde China. 
También es un elemento relevante el diseño de planta, dado que se aprecia capacidad instalada ociosa, probablemente debido a que existe una dispersión de servicios que se ofrece a los clientes. Se requiere considerar los métodos de producción y la disponibilidad de personal adicional temporal, así como el mantenimiento de la maquinaria con personal capacitado, que en conjunto optimizan los procesos.

Un aspecto que se ejecuta en la totalidad de las empresas es la recomendación «boca a boca» para la difusión de su producción, así como el servicio postventa, mediante la instalación, mantenimiento y reparación, el cual es un aspecto clave a resaltar y potenciar.

La gestión de recursos vinculados a factores de calidad y su certificación es un aspecto relevante dentro de la capacidad de innovación expresada en varias mypes. Esto puede expresarse en la adecuación de tecnologías obtenidas de internet, uso de insumos y aprovechamiento de experiencias previas; también estos aspectos pasan a ser relevantes en la adición de valor para las actividades de operaciones cuando contribuyen a la productividad y eficiencia. Si bien se aprecia que el desempeño de las actividades de marketing y ventas suele estar respaldado por la experiencia previa, se debe desarrollar mejoras con relación a los servicios necesarios para trabajar con el cliente, en particular mediante el uso de las tecnologías de información y comunicación (TIC).

\section{Conclusiones}

Las mypes del sector metalmecánico de Arequipa incluidas en la muestra tienen de 6 a 10 años desde su fundación y están dirigidas por hombres adultos. Típicamente son empresas familiares, con prioridades de inversión vinculada a los bienes de capital y de especialización productiva.

En los subsectores de fabricación de hierro y acero, construcciones metálicas, electromecánica y servicios técnico-industriales y producción de máquinas y equipos, se aprecia que los procesos relacionados con las operaciones son fundamentales (diseño, soldadura y montaje). La gestión de proveedores mediante la provisión de insumos a tiempo, así como de los almacenes e inventarios, contribuye directamente al éxito de los procesos productivos. Se aprecia que las operaciones de diseño están más vinculadas a la innovación relacionada con la soldadura y el montaje.

Se observa que es preciso implementar, fortalecer u optimizar el diseño de la planta, el control de la capacidad ociosa y el control en el proceso 
productivo. Asimismo, resalta que es crucial invertir en la certificación de la calidad. Como ventaja, se observa la importante capacidad de innovación para la adecuación de insumos y procesos a la realidad local, así como el servicio postventa o garantía en los productos entregados.

Con el fin de promover la generación de valor en las actividades primarias y de apoyo con mayor desempeño del sector metalmecánico, los resultados sugieren que se requiere fortalecer la difusión de tecnologías, articular los servicios con los proveedores y contribuir a la generación de personal capacitado. Se recomienda a las empresas, academia y gobierno realizar acciones en este sentido.

\section{Referencias bibliográficas}

Cohen, M. y Ballara, G. (2012). La situación de las PyMEs en América Latina. PEAL, 12-30. http://copal.org.ar/ wp-content/uploads/2015/06/peal.pdf

Estrada, R., García, D. y Sánchez, V. (2008). Factores determinantes del éxito competitivo en la Pyme: Estudio Empírico en México. Revista Venezolana de Gerencia, 14(46), 169-182. http://www.redalyc.org/articulo. oa?id=29011523002

García, M. (2012). Una propuesta para la determinación de la competitividad en la Pyme latinoamericana. Pensamiento y gestión, 93-124.

Gereffi, G. (1999). International trade and industrial upgrading in the apparel commodity chain. Journal of International Economics,48(1), 5-29. http://openscienceasap.org/wp-content/uploads/2013/10/ Gereffi_1999_Commodity-chains1.pdf

Grant, R. M. (1996). Dirección Estratégica. Conceptos, técnicas y aplicaciones. Madrid: Cívitas.

Hall, R. (1992). The strategic analysis of intangible resources. Strategic Management Journal, 13, 44-135.

Hamel, G. y Prahalad, C. (1994). Competing for the future. Harvard Business Review, 72(4), 112-128. https://hbr. org/1994/07/competing-for-the-future

Instituto de Desarrollo Industrial Tecnológico y de Servicios. (2005). Plan Estratégico: Sector Metalmecánico. Mendoza: IDITS. http://www.idits.org.ar/Nuevo/Servicios/Publicaciones/SectorMetalmecanico/Plan_ estrategico_sector_metalmecanico-IDITS.pdf

Instituto Nacional de Estadística e Informática [INEI]. (2019). Encuesta Nacional de Hogares ENAHO. https://www. inei.gob.pe/media/MenuRecursivo/publicaciones_digitales/Est/Lib1678/libro.pdf

INEI. (2018). Primer Trimestre. Instituto Nacional de Estadística e Informática. http://m.inei.gob.pe/media/ MenuRecursivo/noticias/nota-de-prensa-n-084-2018-inei.pdf 
Kaplinsky, R. (2000). Spreading the gains from globalization: what can be learned from value chain analysis? Institute of Development Studies, 37(2). https://www.ids.ac.uk/download.php?file=files/Wp110.pdf

Kaplinsky, R. y Morris, M. (2000). A Handbook for Value Chain Research. International Development Research Centre. http://www.fao.org/fileadmin/user_upload/fisheries/docs/Value_Chain_Handbool.pdf

Labarca, N. y García, J. (2011). Elementos para la competitividad en el sector metalmecánico del estado Zulia. Multiciencia, 11(3), 256-261. http://www.redalyc.org/articulo.oa?id=90421736005

Mathison, L., Gándara, J., Primera, C. y García, L. (2007). Innovación: Factor clave para lograr ventajas competitivas. Revista Negotium, 3(7), 65-83. https://www.redalyc.org/pdf/782/78230705.pdf

Mendes de Paula, G. (2015). Importancia económica, inversiones y comercio internacional. Cadena Metalmecánica en América Latina (Alacero), 8-10. https://www.alacero.org/sites/default/files/estudios-estadisticas/cadena_ metalmecanica_en_america_latina._importancia_economica_inversiones_y_comercio_internacional_2015.pdf

Ministerio de Economía y Finanzas [MEF]. (2018). Principales ejes para impulsar la competitividad y productividad. Lima: Ministerio de Economía y Finanzas. https://www.mef.gob.pe/contenidos/archivos-descarga/ competitividad_productividad.pdf

Ministerio de la Producción. (2015). Las Mipymes en cifras 2014. Lima: Ministerio de la Producción.

Ministerio de Trabajo y Promoción del Empleo. (30 de septiembre de 2008). Texto único ordenado de la ley de promoción de la competitividad, formalización y desarrollo de la micro y pequeña empresa y del acceso al empleo decente, Ley MYPE. https://www.sunat.gob.pe/orientacion/mypes/normas/ds0072008.pdf

Nutz, N. y Sievers, M. (2016). Guía General para el Desarrollo de Cadenas de Valor: Cómo crear empleo y mejores condiciones de trabajo en sectores objetivos. Ginebra: Oficina Internacional del Trabajo. https://www. ilo.org/wcmsp5/groups/public/---ed_emp/---emp_ent/---ifp_seed/documents/instructionalmaterial/ wcms_541432.pdf

Paravié, D., Rohvein, C., Urrutia, S., Roark, G. y Ottogalli, D. (2012). Diseño de un instrumento para evaluar el desempeño de las actividades que integran la cadena de valor de pymes metalmecánicas de Olavarría. INGE CUC, 7-32. https://revistascientificas.cuc.edu.co/ingecuc/article/view/221/211

Peteraf, M. (1993). The Cornestones of Competitive Advantage: A Resource - Base View. In Strategic Management Journal, 3, 179-191. https://josephmahoney.web.illinois.edu/BA545_Fall\%202019/ Peteraf\%20(1993).pdf

Porter, M. (1987). Ventaja competitiva. Crecimiento y sostenibilidad de un rendimiento superior. Madrid: Pirámide.

Prahalad, K. y Hamel, G. (1990). The core competence of the corporation. Harvard Business Review, mayo-junio 1990, 79-91.

Programa de Transformación Productiva. (2013). Plan de Negocio para el sector siderúrgico, metalmecánico y astillero. Programa de Transformación Productiva, 71-89.

Saavedra, M. y Milla, S. (2012). La competitividad en el nivel micro de la mipyme en el estado de Querétaro. Querétaro: Congreso Investiga. 
Sociedad Nacional de Industrias [SNI]. (2019). Industria Manufacturera N¹1- noviembre 2019. Estudios Económicos- Reporte Estadístico, 3-5. https://sni.org.pe/no-11-noviembre-2019

SNI. (2020). Reporte Estadístico N 1 enero. https://sni.org.pe/no-01-enero-2020

Superintendencia Nacional de Aduanas y de Administración Tributaria [Sunat]. (19 de marzo de 2019). Nota Tributaria y Aduanera. Contribuyentes inscritos según Actividad Económica, 2005-2020. https://www.sunat. gob.pe/estadisticasestudios/registro-baseTributaria.html

Unión Industrial Argentina. (2013). Debilidades y desafíos tecnológicos del sector productivo. Río Negro: Enciclopedia de Ciencias y Tecnologías en Argentina ECYT-AR. http://cyt-ar.com.ar/cyt-ar/images/f/f8/ Debilidades_y_desaf\%C3\%ADos_tecnol\%C3\%B3gicos_del_sector_productivo.pdf

Wernerfelt, B. (1984). A resource-based view of the firm. Strategic Management Journal, 5(2), 171-180. http:// web.mit.edu/bwerner/www/papers/AResource-BasedViewoftheFirm.pdf 